\title{
Less Than or Equal To
}

National Cancer Institute

\section{Source}

National Cancer Institute. Less Than or Equal To. NCI Thesaurus. Code C61586.

A statement about the relative size or order of two objects specifying that either both objects are the same in quantity or measure or value or status or that the object of interest is smaller than the other object in one of these ways. 